\title{
Erratum: Electrically switchable surface waves and bouncing droplets excited on a liquid metal bath [Phys. Rev. Fluids 3, 124804 (2018)]
}

\author{
Xi Zhao $\odot$, Jianbo Tang $®$, and Jing Liu \\ Q (Received 20 July 2021; published 5 August 2021)
}

DOI: 10.1103/PhysRevFluids.6.089901

During our ongoing experiments, we noticed a calibration issue with the acceleration measurement in our system which led to acceleration amplitudes ten times higher than the actual values. Consequently, the experimentally measured driving acceleration amplitude $a$, the normalized acceleration $\Gamma$ including also $\Delta \Gamma$ and the threshold values $\Gamma_{X}(X=B, C, D, E, F$, and $P D B)$, and the coefficient $C_{0}$ in Eq. (1) should be multiplied by a factor of 0.1 . The viscosity of the $\mathrm{NaOH}$ electrolyte $(0.25 \mathrm{~mol} / \mathrm{l})$ should be $v_{E}=9.4 \times 10^{-7} \mathrm{~m}^{2} / \mathrm{s}$.

This correction applies to Figs. 2, 3, 4, 5, and 6, and Eq. (1).

This error does not affect the results and conclusions of the article. 